\title{
The Research on the Electronic Blue Army Exercise of Combat Teaching Model
}

\author{
Liang Ma \\ Department of Surface Ship Command, Dalian Naval Academy, Dalian, 116018, China \\ 446982051@qq.com
}

Keywords: Electronic blues; The combat; Comprehensive practice; Teaching mode

\begin{abstract}
The introduction of electronic in the process of practical teaching system of the blues comprehensive exercise is an effective method for checking our fighting training methods. Expounds the electronic blues combat exercise their own training and against the two modes of training, put forward the method based on ACP electronic blues combat exercise strategy analysis frame, set up electronic blues combat exercise strategy analysis of parallel systems, studies the comprehensive practice to organize the implementation of the combat teaching method, based on the electronic blues comprehensive practice provides a new way of researching the combat teaching mode.
\end{abstract}

\section{Introduction}

Electronic blues is an important part of the blues' system, based on informatization trained troops major combat simulation object, the collection of electronic attack, defense electronics, electronic support, and many other factors in the integration of electronic information simulated combat power. Electronic blues is able to realistically simulate combat against tactical ideas, methods, battlefield environment, the tactics for the weapon equipment operational links such as performance and operational elements, in strategy, tactics, weapons, technology, electromagnetic, etc to provide their own combat against test, training practice environment and drills. By studying the electronic blues tactical ideas, thus pulling our methods developed to the direction of the combat teaching mode innovation[1].

\section{Electronic Blues Combat Exercise Demand Analysis}

Electronic Blues Combat Exercise Mode. Electronic blues combat exercise mode the elements including command, weapon equipment system, simulated electronic countermeasure combat power and comprehensive security four aspects, can be used in training exercises, and operational test equipment, electronic countermeasures methods innovation, etc. Electronic blues refers to the combat comprehensive exercise, according to the scenario, integrated multiple electronic blues or or more of the subjects of the red army equipment training content coherence of exercise. Purpose is to make through drills force familiar with related equipment technical performance, system characteristic, tactical methods and operational application, clear equipment into the system, the position and role of the operational system, lay a foundation to give full play to the equipment operational effectiveness. Electronic blues combat exercise including their own training and combat application 2 pattern. The combat exercise mode as shown in Fig. 1 


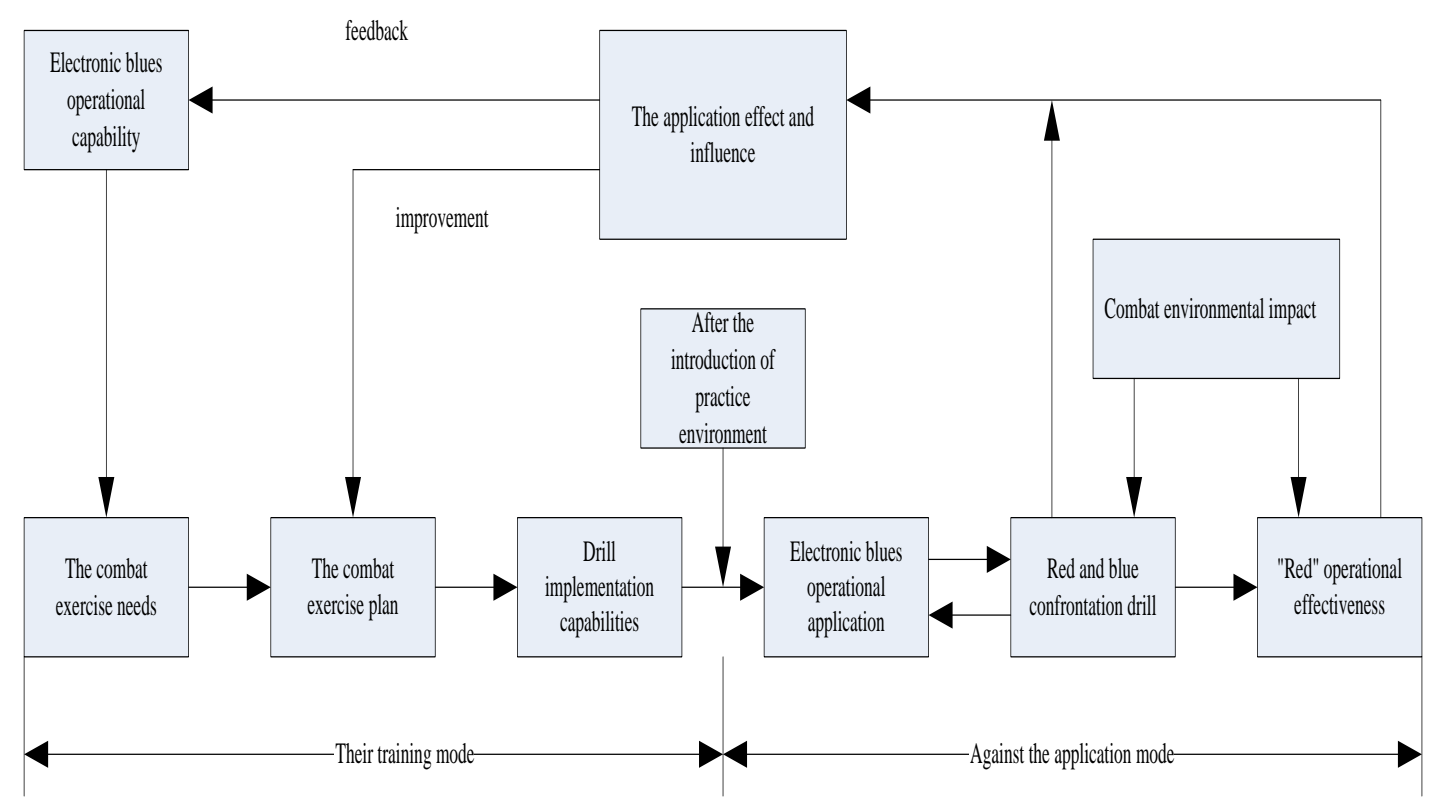

Figure 1. electronic the blues and the relationship between the combat exercise mode

(1) Their own training mode. To improve the simulated combat ability to implement their own electronic blues combat comprehensive practice activity. Their training mode is the advanced stage of electronic blues equipment training. Main purpose is: exercise increases the blues command organs, commander rival organization simulation operation command and wartime business level; Exercise increases the blues each unit simulation battle rival carries out all kinds of electronic countermeasures operations and combat mission equipment technical skills; Exercise increases the blues closely cooperate with each unit coordinated system capabilities; Exercise increases the blues equipment operational application level.

(2) Against the application model. It is using electronic the blues as fighting competitors participate in military training exercises on the implementation of tasks such as the combat comprehensive practice activity. Against the application mode of electronic supporting the blues as a comprehensive exercise by acting as "enemy" according to the scenario guide to participate in military training exercise, and operational test equipment, and electronic countermeasures methods innovation of the combat exercise. Main purpose is to provide realistic task of training exercises the army combat against environment and combat. Against the application mode of electronic blues combat exercise plan, implement and guide command and assessment based on the content of the red army related tasks.

Electronic Blues Combat Exercise Strategy Analysis and Features. Electronic blues combat exercise strategy analysis of the optimizing decision support as the goal, application based on the quantitative analysis of the scientific method for electronic blues combat exercise about military decision making problems for research or knowledge. Its purpose is to deepen the cognition of the blues in the electronic construction characteristics and laws, support the implementation of each command management or organization hierarchy decision, improve electronic blues equipment in complex electromagnetic environment adaptability and simulation capabilities (system). Strategy analysis mainly includes the blues in the electronic construction demonstration, exercises demand analysis, scheme optimization, guide and command decision aid and five field exercise effect evaluation. 
(1) Electronic blues construction demonstration. The blues in the electronic construction is a complex systems engineering, and construction of multi-functional total factor requires high cost should be based on the current state and future strategic direction key areas for construction. According to the characteristics of joint operations and services and arms forces combat mission requirements and overall planning. Construction of electronic Chelsea's main aim is to friendly forces training exercises, and operational test equipment, and electronic countermeasures methods innovation tasks such as providing realistic combat, promote the development of their own new combat power and the ability to combat troops system generated. Strategy analysis theory and method, demonstrates the blues in the electronic construction key, controllable factors, uncontrollable factors, environmental factors, etc. For the blues in the electronic construction scheme optimization and decision-making to provide the reference basis.

(2) Comprehensive demand analysis. Clear demand is electronic blues combat exercise the basis of organizing the implementation. It decides whether to carry out the target and carried out in each task and task requirements. In the background of practice task based, resources, environment, conditions, existing problems and possible solutions on the basis of the preliminary analysis, emphasis whether each mission objectives and projects with the blues capabilities, electronic confrontation forces combat training exercise needs consistent. Drills demand management analysis results can provide electronic blues combat exercise the formulation and adjustment of mission, goals, provide a reference or guide.

(3) Comprehensive scheme optimization. Integrated scheme is to achieve comprehensive practice on electronic blues replays all the work of the organization and implement comprehensive exercises to do detailed planning and arrangement. Mainly includes the implementation plan, evaluation and protection plan. Implementation plan is the overall planning and arrangement of the implementation of practice is the fundamental basis for the implementation of practice. Is the specification and guidance practice activities; Assessment plan is to evaluate exercise information collection and analysis of the specific planning and arrangement is the main basis of information collection and evaluation personnel work. Security program to ensure the smooth implementation and exercise plan of all. Including: display, alarm service support (drill area control), technical support, material equipment support, etc. Scheme optimization mainly aims at the feasibility and effectiveness of comprehensive practice plan for can reach the target, on how to plan and procedures to meet the demand, resource allocation optimization and decision support.

(4) Guide and command decision aid. Scientific, effective, timely, standardized guide with the successful completion of the command decision-making is electronic blues the task of the combat exercise important basis and prerequisite. Electronic blues with new equipment technology, strong systemic and platform diversity, makes the blues combat exercise guide and command decisions harder, cannot use a single command way against all practice task, it is impossible to charge only a way of directing the whole practice process, is more impossible to use other types of equipment drill way of command. Guide adjustment and command decisions throughout the electronics blues combat the whole process of comprehensive practice tasks, organization and planning, coordinating control exercise is the key to successful completion. How to auxiliary guide attune and command decision is electronic blues combat exercise strategy analysis need to solve key problems[2].

(5) Comprehensive evaluation. Comprehensive evaluation strategy analysis is not only to all and to assess the blues forces in the equipment operation simulation, 
electronic confrontation tactics application simulation, simulation and command systems and exercise effect. And systematic analysis of the electronic blues combat exercise theory, organization method, guide method, comprehensive evaluation method, the method of building the blues, security and so on various aspects and performance, the effect of objective and accurate comprehensive evaluation conclusion, the research results and practical significant exercise, provides reference for the further development of comprehensive practice or reference.

\section{Construction of Electronic Blues Exercise System}

Based on Strategy Analysis Frame of ACP Method. Parallel system is to point to by a natural and corresponding to one or more virtual reality system or an ideal artificial system composed of common system. Its main purpose is through the actual system and the artificial systems are connected to the trainer behavior between the comparison and analysis, complete conditions for their future "reference", "forecast" and "regulation", etc. Electronic blues actual system combined with artificial systems, electronic blues combat exercise can be rolling iterative strategy analysis problem analysis, solving and optimizing, especially various cannot in live-fire conditions of the real situation setting, fault, or battle scenes, or in the electronic blues combat exercise practice, take a long time to finish the task can be in electronic blues artificial system is low cost, no risk to run efficiently. Based on the method of ACP strategy analysis frame is shown in Fig. 2.

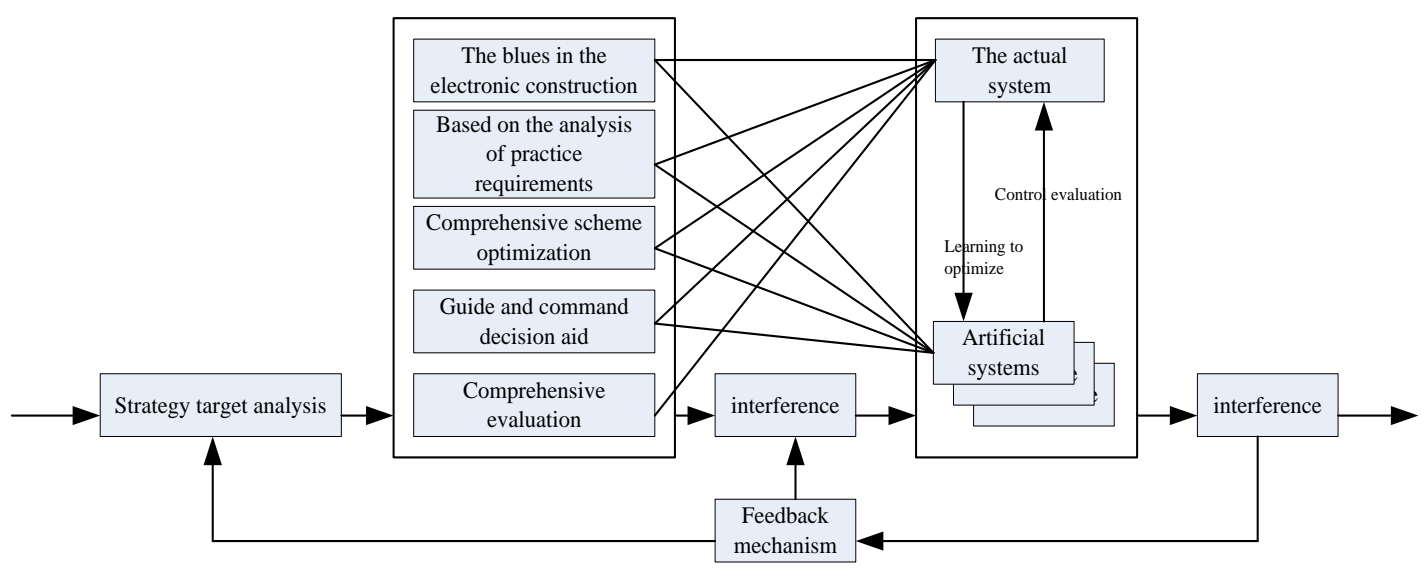

Figure 2. ACP method based electronic blues combat exercise strategy analysis frame

Build Strategy Analysis of Parallel System. Strategy analysis of parallel system under the framework of AC 'P method system by actual combat system, artificial system of 2 parts. Actual combat system refers to the electronic system and function of the blues warfare. Including physical, modeling and simulation system, it is electronic blues in the main body of the combat exercise, electronic blues strategy analysis target and the object of the research; Artificial combat system is established to solve the problem of electronic blues strategy analysis of electronic blues combat simulation system, the electronic blues strategy analysis methods and means of the research. 


\section{Exercise the Combat Teaching Organization and Implementation}

Comprehensive practice committee shall, according to the intention of the comprehensive practice always guide, around training objects and training purpose, to guide the deduction, its work procedure and main content includes:

(1) Release exercise supplement

Comprehensive practice at the start of the deduction, comprehensive exercises to the steering group command post (integrated practice marshalling) announced began to exercise a posteriori operational time, publish supplement, comprehensive exercise personnel enter the deduction, induction formation situation; Operational time usually determine according to the need of exercise plan and resolve scheme. The content of the supplement is usually include: to the current operational time, about politics, diplomacy, military, etc; The possibility of conflict and conflict results; Through a variety of reconnaissance troops to deploy and found activity; The current operational area over a period of time's weather forecast, etc.

(2) Guide

After entering deduction status, exercise steering group shall, according to the determination of battlefield situation and the comprehensive practice and measures, timely advance time, release the plot, subject, content and training as planned exercise induced personnel to enter; Can continuous operational time, also can be intermittent, advance ratio is usually based on the comprehensive practice exercise, contents, methods, personnel training level and different stages of comprehensive practice deduction to determine[4].

(3) Release the plot

Comprehensive practice guide in the form of release various plot usually adjustable steering group exercise personnel; Release the plot revolves around the training focus, content variety, reasonable and conforms to the battlefield, close to actual combat, strictly avoid simplistic plot and modelling; General plot in each group should have operational circumstances and logistics equipment support circumstances, timely release political work plot, make exercise researchers get comprehensive training; Plot format should be standard, content is practical, often in bulletin, orders, directives and approval, the report form.

(4) Comprehensive practice self-care

Comprehensive practice after steering group released the plot, it shall timely communicate intentions to regulate member and regulate member on this basis, according to the provisions of self-care.

(5) Exercise

When confrontational comprehensive exercises, for both red and blue force action conflicts, comprehensive exercises should be based on both sides of the steering group decision, determination, and measures and combining with the current deduction, will fight ruling result into a new situation in time, to give both sides as a condition and comprehensive drills a posteriori, continue to guide the deduction; Decision shall, in accordance with the principle of fighting, usually when combat operations (data), considering the influence of factors such as terrain, weather, and the pros and cons of the two sides resolve to disposal and forces act to correct; When using a computer to add ruling, comprehensive exercises steering group can according to need, organization conflict decision on site.

(6) Disposal

In comprehensive practice exercise, exercise the situation of the steering group on comprehensive drilling personnel disposal are generally not direct intervention, but when exercise researchers a significant error in determination or measures, or the two 
sides in the antagonistic exercise action forces conflicts affect the comprehensive practice process or the result of the training effect, the comprehensive practice committee shall, according to the comprehensive practice always guide's instructions, induction of both sides to take remedial measures, or directly to the identity of both the superior to disposal of some cases, to ensure continued to exercise deduction; When deduction at some stage to achieve training or training question is no exercise, with the approval of the comprehensive practice always guidance, comprehensive practice visual sentiment deduction plan adjustment, steering group to ensure that focus on the practice of the training content; To some key problems, according to the need of comprehensive practice adopt the method of withdrawal of comprehensive practice, organization guide personnel to conduct workshops, unified thought, continue to exercise again into the situation.

(7) Organization integrated practice summarized and discussed

Disposal of decision-making on comprehensive practice personnel to complete each step, comprehensive practice committee shall timely organize exercise personnel to discuss, to inspire each other, and to prepare for the unified disposition decision. Discussion, the comprehensive practice guidance personnel shall be conducted in a timely manner to guide, to promote the development of further research problems, and to grasp the direction of the comprehensive practice discussion.

(8)Comprehensive practice summarizing assessment and evaluation

Due to the comprehensive practice field exercises take way, around the mission, in the practical environment, according to the actual combat scheme plan, the whole system, total factor, the whole process of comprehensive training. Comprehensive practice through the whole process of comprehensive evaluation assessment, therefore, not only can find and solve the contract tactical unit in carries out combat missions have exposed weaknesses, important and difficult problem, more to the combat methods innovation and system capabilities. At this stage the faculty organization students to evaluation of the comprehensive practice stage, to the scheme of artificial, to red and blue summarized the condition of confrontation, at this stage it insert link according to the content of the evaluation guide students deepen the understanding of the methods, application.

\section{References}

[1] Galay Barbarosoglu, Linet Ozdamar, Ahmel Cevik. An Interactive Appmach of Hierarchical Analysis of Helicopter Logistics in Disaster Relief Operations [J]. European Journal of Operational Research, 2002: 118-133

[2] Omid Shakernia. Vision-based Control and Coordination of Unmanned Vehicles[D].California: University of California, Berkely 2003

[3] Sriram Venkataramanan, Atilla Dogan.A Multi-UAV Simulation for Formation Reconfiguration[R]. AIAA2004-4800. Providence, Rhode Island: AIAA Modeling and Simulation Technologies Conference and Exhibit,2003

[4] Donald W.Manned/unmanned common architecture program (MCAP): a review[C]// Proceed--ings of the22nd Digital Avionics Systems Conference.2003: 6.B.4/1.6.B.4/7.

[5] M.Valenti, T.Schouwenaars, Y.Kuwata,E.Feron and J. How Implementation of a Manned Vehicle-UAV Mission System[C].AAIA Guidance, Navigation and control Conference, Providence, RI, August 2004,AIAA2004 
[6] Sarit Kraus.Negotiation and cooperation in mulfi-ageat environments.Artificial Intelligence.1997: 79-97

[7] M.Ben-Bassat. Knowledge Requirement and Management in Expert Decision Support Systems for (Military) Situation Assessment[J]. IEEE Trans. on SMC.1982, 12(4):479-490

[8] Jerome Azarewicz, Glenn Fala. Template-based Multi-agent Plan Recognition for Tactical Situation Assessment[A].In: Proceedings of 5th conference on Artificial Intelligence Applications[C], March 1989:247-254

[9] G.Peterson, L.Axelsson, T.Jensen, etal. Multi-source Integration and Temporal Situation Assessment in Air Combat[J]. Proceedings of Information Decision and Control.1999:371-375

[10]Ge S S,Cui Y J.Dynamic motion planning for mobile robots using protential field method[J].Autonomous Robots,2002,13:207-222 